\title{
La continuità assistenziale nella gestione delle lesioni da pressione: un opuscolo informativo per la collaborazione ospedale-territorio
}

\author{
Margherita Farí, ${ }^{1}$ Maurizio Mercuri, ${ }^{2}$ Alessandro Scalise ${ }^{3}$ \\ ${ }^{1}$ Facoltà di Medicina e Chirurgia Università Politecnica delle Marche, infermieristica; ${ }^{2}$ Facoltà di Medicina e Chirurgia Università Politecnica \\ delle Marche, infermieristica; ${ }^{3}$ Clinica di Chirurgia Plastica e Ricostruttiva, Università Politecnica delle Marche, Ancona, Italia
}

\section{RIASSUNTO}

Il progetto consiste nella creazione di un opuscolo informativo, all'interno del quale sono spiegate le principali informazioni relative la gestione del paziente portatore di Lesioni da Pressione. Le nozioni espresse sono alla base del percorso terapeutico, in quando sono indirizzate a lettori non esperti nel campo delle medicazioni difficili. Viste le esigenze del pubblico di assistere a questa tipologia di pazienti, in molti casi in maniera autonoma per quanto riguarda la medicazione vera e proprio, si è ritenuto necessario fornire del materiale per aumentare le conoscenze dei caregiver informali. Lo scopo ultimo è proprio quello di fornire un metodo semplice e sicuro di apprendimento per i cittadini che si occupano della gestione del paziente e delle relative medicazioni; considerando sempre un affiancamento da parte di professionisti. Gli obiettivi che ci si pone con il progetto sono: valutare e visualizzare quelli che sono gli aspetti importanti per i soggetti aventi Lesioni da Pressione, questo comprende la definizione, prevenzione, classificazione e trattamento; l'importanza della continuità assistenziale per questa tipologia di paziente; informare il cittadino su cosa sono le lesioni da pressione (quali sono le accortezze quotidiane da dover attuare per evitare il peggioramento o lo stallo della condizione) ed infine il trattamento vero e proprio tramite l'insegnamento delle medicazioni difficili e della loro applicazione in base ai diversi casi (essendo un argomento vasto e materia di specializzazione in ambito infermieristico si tratterà in maniera semplificata in modo da dare informazioni basilari ma utili). Per la creazione dell'opuscolo è stata effettuata una revisione della letteratura che vede la consultazione di due linee guida, undici articoli e due testi.

\section{INTRODUZIONE}

Le Lesioni Da Pressione (LDP) sono un fenomeno molto diffuso dato dall'aumento dei fattori che portano al

Corrispondenza: Margherita Farí, Facoltà di Medicina e Chirurgia, Università Politecnica delle Marche. Via Tronto 10, Ancona, Italia

E-mail: margherita_fari@libero.it

Parole chiave: Trattamento lesioni da pressione; medicazioni avanzate; lesioni da pressione; preparazione del letto della ferita; caregiver.

Conflitto di interessi: Gli autori dichiarano l'assenza di conflitto di interessi.

Disponibilità di dati e materiali: I dati analizzati in questo articolo sono disponibili su richiesta.

Approvazione etica e consenso alla partecipazione: Non applicabile.

Ricevuto per la pubblicazione: 20 luglio 2020.

Accettato per la pubblicazione: 14 dicembre 2020.

This work is licensed under a Creative Commons Attribution NonCommercial 4.0 License (CC BY-NC 4.0).

${ }^{\circ}$ Copyright: the Author(s), 2021

Licensee PAGEPress, Italy

Italian Journal of Wound Care 2021; 5(1):65

doi:10.4081/ijwc.2021.65 loro sviluppo. Facendo un'analisi più in generale, si può affermare che i pazienti portatori di LDP fanno parte della moltitudine di soggetti affetti da condizioni croniche che in Italia sono patologie in crescita poiché correlate all'aumento dell'età media.

È stato visto, infatti, che le patologie croniche sono più frequenti nelle fasce d'età adulta, comprese tra 55-59 anni. Il $54.1 \%$ dei soggetti soffre di una condizione cronica, cosi come con l'aumentare dell'età notiamo anche l'aumento della percentuale, infatti sopra i 75 anni la percentuale aumenta del $86.9 \%$ (vedi Figura 1 ). ${ }^{1}$

La particolarità delle LDP è che, oltre a fare parte di quelle che vengono considerate patologie croniche, vengono molto spesso generate da condizioni croniche che comportano la sedentarietà del soggetto e uno stato nutrizionale non ottimale, entrambi fattori molto influenti nella formazione di queste particolari lesioni.

Le malattie a lento decorso non vengono più trattate completamente in ospedale per diversi motivi legati sia alla modalità di guarigione migliore per il paziente, sia al costo che comporterebbe per l'azienda.

È stato visto che una degenza prolungata, molto spesso superflua, comporta l'aggravarsi delle condizioni psico-fisiche del paziente, che si trova ad affrontare la sua malattia in un ambiente a lui sconosciuto e lontano dagli affetti familiari.

Si preferisce affrontare con il paziente un percorso che vede in primo luogo l'attivazione del territorio e di tutte quelle che vengono considerate le cure domiciliari. Queste, 
infatti, forniscono la possibilità di erogare a domicilio servizi che contribuiscono al mantenimento del massimo livello di benessere.

Un altro aspetto positivo del trattare il paziente in strutture e luoghi diversi da quello ospedaliero è l'aumento del coping da parte del soggetto stesso. Il termine indica 'l'insieme dei meccanismi psicologici adattativi messi in atto da un individuo per fronteggiare problemi emotivi ed interpersonali, allo scopo di gestire, ridurre o tollerare lo stress ed il conflitto."

Dall'altra parte invece troviamo una conseguenza economica per l'azienda stessa; se ogni soggetto affetto da patologie croniche venisse trattato in ospedale, fino alla risoluzione della condizione, le spese avrebbero un peso importante all'interno del bilancio aziendale, che ne risentirebbe modo significativo.

Per questo motivo ad oggi possiamo dire che in ospedale vengono trattate principalmente le condizioni acute mentre a livello territoriale vengono trattate le condizioni croniche.

Queste due realtà non dovrebbero essere considerate distinte tra loro, in quanto invece esse entrano costantemente in relazione e ciò garantisce il miglior trattamento per il paziente. $^{3}$

In particolar modo, per quanto riguarda i soggetti affetti da LDP, questi vengono visitati periodicamente in centri specializzati per il trattamento delle ferite difficili e, in alcuni casi, vengono trattati anche tramite intervento chirurgico, sempre all'interno dell'azienda ospedaliera. Per quanto riguarda la gestione quotidiana della lesione, invece, questa viene affidata a un caregiver che può essere sia un professionista che opera a livello territoriale, sia un conoscente o un parente a cui si affida la gestione del paziente.

È importante quindi assicurare un'ottima collaborazione tra ospedale e territorio per poter garantire al paziente il miglior trattamento e la risoluzione del problema nel minor tempo possibile.

Per quanto riguarda quelli che sono i caregiver informali, ovvero tutti quei soggetti a cui viene affidato il paziente e che non hanno le conoscenze e competenze in materia, è importante fornire loro uno strumento semplice e sicuro d'informazione per l'applicazione di quelle che saranno le medicazioni ottimali. Non solo: dovranno riuscire a gestire tutte le accortezze da attuare con un paziente affetto da LDP.

Per questo motivo è stato progettato un opuscolo informativo al fine d'informare su quelle che sono le nozioni base per la miglior assistenza e di conseguenza agevolare anche quello che sarà il lavoro del team ospedaliero.

\section{MATERIALI E METODI}

L'opuscolo informativo che vorremmo realizzare ha come obiettivo principale quello di valutare e visualizzare gli aspetti importanti per i soggetti portatori di LDP. Il contenuto dell'opuscolo comprenderà nozioni riguardo: la definizione, prevenzione, classificazione e trattamento delle LDP; l'importanza della continuità assistenziale per questa tipologia di paziente; spiegazioni sulle accortezze quotidiane da dover attuare per evitare il peggioramento o la cronicizzazione delle lesioni; il trattamento vero e proprio tramite l'insegnamento delle metodiche di medicazione e della applicazione di medicazioni avanzate diverse in base allo stadio delle lesioni. ${ }^{4}$ Naturalmente, essendo questo del wound care un argomento vasto e materia di specializzazione in ambito infermieristico, si tratterà in maniera semplificata in modo da dare nozioni basilari ma utili e comprensibili.

Il tutto viene svolto con la prospettiva di attuare un percorso caratterizzato dalla continuità assistenziale, evitando che il piano terapeutico si svolga esclusivamente nelle strutture ospedaliere, ma invitando il cittadino ad essere anche esso parte attiva di un processo che vede come elemento centrale il paziente. Il lavoro concreto su cui si basa questo progetto mira proprio al coinvolgimento dei caregiver in modo che essi possano attuare tutte quelle procedure che formano la continuità nel piano assistenziale del singolo paziente.

Ma cos'è la Continuità Assistenziale? Per spiegare bene il significato di questa attività occorre prima fare alcune considerazioni statistiche.

L'invecchiamento della popolazione è un fenomeno che vede farsi sempre più spazio all'interno dei paesi sviluppati come il nostro. Questo fenomeno comporta la sempre maggior richiesta, da parte del pubblico, dell'assistenza, che comporta la necessità di modificare i modelli organizzativi-gestionali odierni.

Si stima che la fascia di età considerata anziana, e quindi più incline a condizioni croniche, arriverà nel 2020 ad occupare uno spazio all'interno della società pari al

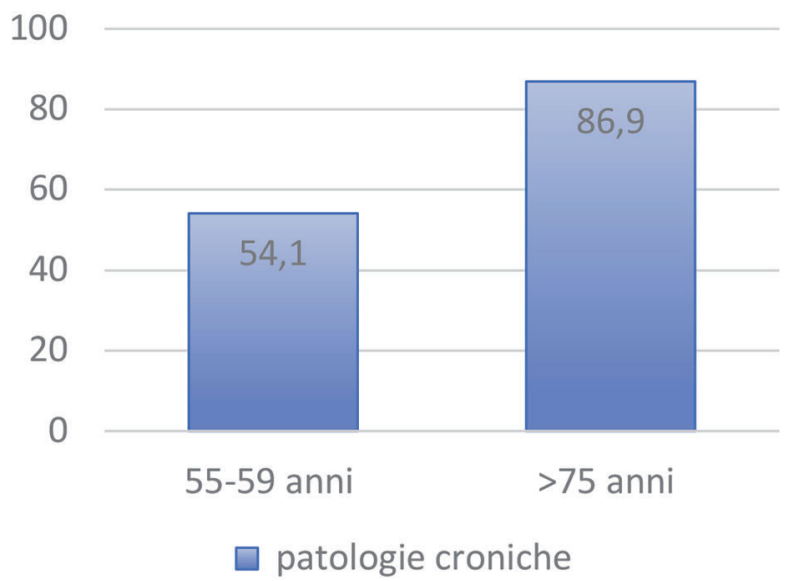

Figura 1. Distribuzione delle patologie croniche nelle fasce di età. 
$23.2 \%$ e che nel 2050 raggiungerà il 33.00\% (vedi Figura 2). I dati ci fanno capire che, con l'aumentare della popolazione considerata più a rischio di sviluppare patologie croniche, anche la richiesta di servizi aumenterà in correlazione.

Per questo motivo, lo stato, già con il L.R. 19/2006 e nel successivo Regolamento Attuativo n. 4/2007, ${ }^{6}$ aveva istituito: la Porta Unica di Accesso (PUA) e l'Unità di Valutazione Multidimensionale (UVM).

La PUA è l'ente al quale il cittadino può rivolgersi per richiedere informazioni sui propri diritti riguardo la possibilità di servizi e interventi a livello locale; l'UVM è formata da un team multidisciplinare, che ha il compito di analizzare la richiesta, fatta dal cittadino, per poi valutare l'effettiva necessità degli interventi. In seguito alle valutazioni fatte, e alla conferma dell'effettiva necessità, verranno attivati i servizi richiesti a favore del cittadino.

Ad oggi la gestione del paziente affetto da condizioni croniche è legata principalmente all'assistenza fornita a li-

\section{POPOLAZIONE NEL 2020}

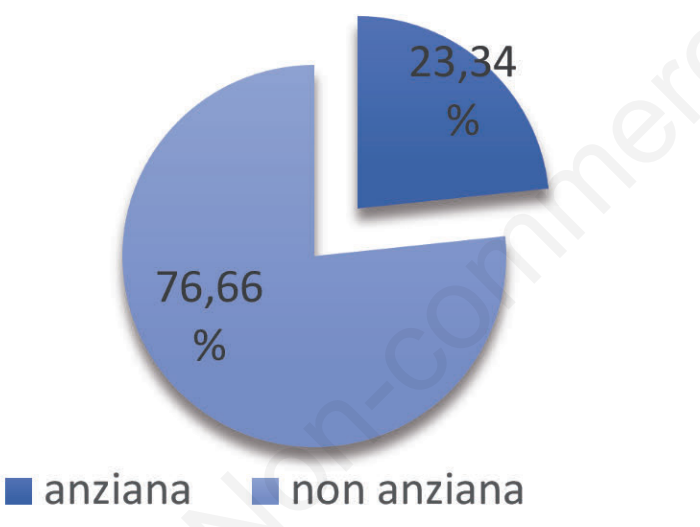

POPOLAZIONE NEL 2050

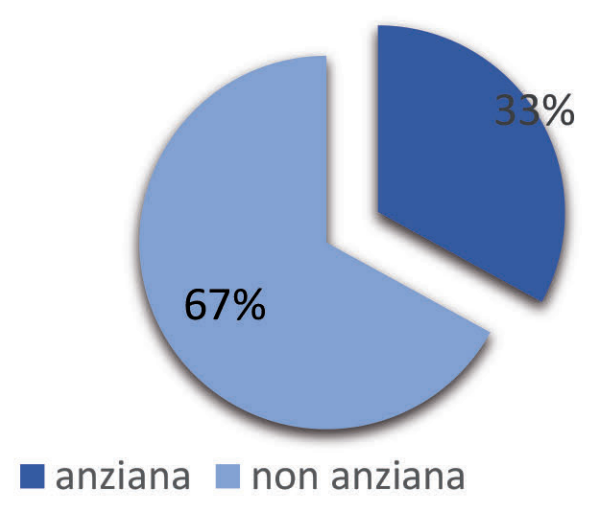

Figura 2. Percentuale della popolazione anziana sul totale. vello territoriale; questa scelta è spinta dalla presenza di una sempre maggior fetta della popolazione affetta da patologie croniche, che non può essere trattata all'interno delle aziende ospedaliere, sia per una questione economica, che per la miglior scelta assistenziale per il paziente.

La spesa che comporterebbe, la gestione di tutti i pazienti cronici all'interno dell'ospedale, sarebbe troppo elevata e andrebbe ad inficiare in modo negativo sulle risorse a disposizione. Per questo motivo ad oggi si adotta una politica che vede la gestione del paziente acuto all'interno dell'ospedale, mentre le condizioni croniche vengono trattate a livello territoriale.

Per quanto riguarda il paziente, un'assistenza prolungata all'interno dell'ospedale sarebbe sconveniente, in quanto la degenza sarebbe lunga e superflua. Si è propensi a trattare il cittadino in un luogo conosciuto, circondato dagli affetti famigliari e seguito da un team di esperti che operano tramite i servizi forniti dal territorio.

Viste le seguenti considerazioni è importante quindi focalizzarsi su quella che viene definita "continuità assistenziale", cioè l'attuazione di servizi messi in atto da professionisti in alternativa alle strutture ospedaliere. L'obiettivo è quello di garantire una continuità dei servizi per il paziente al di fuori dell'azienda, favorendo la progressione nel precorso terapeutico.

\section{DISCUSSIONE}

Le LDP rappresentano appieno un'entità clinica esemplare delle patologie croniche di cui soffre la popolazione. Il trattamento di quest'ultime non vede una prerogativa esclusiva dell'azienda ma, anzi, la maggior parte del lavoro pratico, viene svolto tramite l'attivazione dei servizi territoriali.

Il percorso assistenziale delle LDP prevede il coinvolgimento di differenti figure professionali ed assistenziali: il team di professionisti operanti all'interno della struttura ospedaliera, il team di professionisti operanti a livello territoriale ed i caregiver che, nel loro ruolo, possono essere o no competenti in materia.

Il percorso per questa tipologia di paziente ha inizio con la presa in carico da parte di professionisti che operano all'interno dell'azienda Ospedaliera. Questi analizzano la condizione e la storia clinica per individuare $i$ fattori di rischio presenti, quelli evolutivi ed individuare quali potrebbero ostacolare il percorso terapeutico. L'azienda, quindi, offre come servizio una visione periodica del paziente che mira e visualizzare l'andamento clinico dopo un lasso di tempo più o meno breve a seconda dei casi e delle situazioni. Durante questo incontro viene stabilito il tipo di medicazione da utilizzare e con quale tempistica essa dovrebbe essere eseguita, stabilendo così l'inizio del percorso terapeutico.

Successivamente il cittadino potrà rispettare la prescri- 
zione data in autonomia o usufruendo dei servizi resi disponibili dal territorio, tramite l'attivazione della PUA e della UVM. Nel caso in cui si riscontrasse l'effettiva necessità di un professionista nell'attuazione delle procedure prescritte, il territorio si pone l'obiettivo di elargire un'assistenza periodica assicurata da un professionista (ADI). Quest'ultimo, recandosi a domicilio del paziente con le tempistiche prestabilite, effettuerà le medicazioni in collaborazione e accordo con il team ospedaliero.

Se non si dovesse riscontrare l'effettiva necessità dell'attivazione di un professionista allora sarà il caregiver ad effettuare le medicazioni richieste seguendo le indicazioni date dal team di professionisti.

Nella catena del percorso terapeutico alcuni soggetti, a cui è data la responsabilità della gestione del paziente, non hanno competenze per i trattamenti terapeutici e potrebbero trovarsi di fronte ad una situazione clinica complessa. Per questo motivo è importante educare il cittadino e formarlo riguardo le nozioni base della materia, per evitare che si crei un'interruzione nella catena della continuità assistenziale, fondamentale per la risoluzione della condizione.

Una strada alternativa, rispetto a quella appena enunciata, vede l'attivazione diretta del territorio senza il coinvolgimento in primis dell'azienda ospedaliera. In questo caso professionisti che operano a livello territoriale, come nel caso degli infermieri esperti in wound care, ${ }^{7}$ prestano $\mathrm{i}$ propri servizi nei confronti del paziente a livello domiciliare in autonomia.

È importante sottolineare che in quest'ultimo caso il professionista potrà sempre rivolgersi ad esperti che operano all'interno dell'ospedale, per avere un confronto e per analizzare, tramite un team multidisciplinare, il caso clinico, ed insieme scegliere le strategie terapeutiche migliori per la risoluzione della condizione.

È quindi fondamentale rimarcare l'importanza che ha la continuità assistenziale per questa tipologia di pazienti, che vedono la maggior parte del lavoro svolgersi al di fuori dell'ospedale e in collaborane con figure esperte e non.

L'intento di questo lavoro è quello di fornire materiale informativo a tutti i soggetti non professionisti che hanno in carico un paziente affetto da LDP.

L'opuscolo è stato creato con il fine d'informare il cittadino su quelli che sono gli aspetti generali e gestionali della patologia (vedi Figura 3 ). ${ }^{8}$

A molti di questi soggetti è affidata la gestione di un paziente portatore di LDP e, molto spesso, si trovano di fronte a una condizione che non conoscono.

L'opuscolo è formato da trenta pagine, all'interno delle quali sono state spiegate, in modo semplificato, tutte le nozioni base necessarie per la risoluzione della condizione. ${ }^{9}$

All'interno è spiegato il perché la gestione del paziente non viene affidata esclusivamente all'ospedale e si ha la necessità della loro collaborazione nel percorso terapeutico.

Nelle prime pagine sono introdotte le nozioni base per la vita quotidiana, tra cui la mobilizzazione, l'alimentazione e la cura della pelle. Questi particolari, per quanto possano apparire scontati, sono in realtà alla base del processo di guarigione, e non devono essere trascurati (vedi Figura 4).

La definizione specifica di Lesione da Pressione è stata inserita con la consapevolezza che alcuni termini possono risultare troppo specifici per l'utente ma anche per mantenere un linguaggio scientifico comprensibile a tutti. Non si preclude che alcuni dei soggetti a cui è destinato l'opuscolo siano professionisti che hanno dimestichezza con la materia.

All'interno sono state elencate le classificazioni delle lesioni con annesse immagini, per cercare di facilitare il cittadino nel riconoscimento della lesione, in quanto abbiamo ritenuto che la sola spiegazione sarebbe potuta sembrare complicata al lettore, vista la presenza di termini specifici, essenziali per la classificazione. ${ }^{10}$

Sono presenti scale di valutazione, quali Braden e Push tool (vedi Figura 5). ${ }^{11}$

Quest'ultime sono state inserite per agevolare il lavoro dei professionisti. Se viene effettuata una valutazione regolare dal cittadino si potrà valutare l'andamento periodico fornito da dati certi.

Si potrà basare il piano terapeutico e i suoi cambiamenti su quelli che sono dati oggettivi rilevati dai caregiver. Di conseguenza la valutazione non verrà effettuata solo il giorno dell'incontro con gli esperti ma si potrà valutare un percorso continuativo, che riguarda sia le condizioni generali del paziente, con i suoi relativi fattori di rischio (Braden), sia l'andamento della ferita (Push Tool).

L'acronimo T.I.M.E. ${ }^{12}$ è stato inserito perché è un passaggio fondamentale nella gestione del paziente, ma non ci si aspetta che il cittadino conosca tutte le nozioni inerenti a questa fase. L'acronimo è correlato di relativa definizione ad ogni lettera per semplificare la lettura anche ai non esperti in modo che l'utente ne conosca il significato.

La parte più concreta del progetto consiste nell'introdurre tramite tabelle tutte le medicazioni avanzate considerate più idonee, per i singoli stadi (vedi Figura 6). ${ }^{13}$

Il professionista dopo valutazione della lesione avrà il compito di indicare la medicazione più adatta tra quelle proposte. Al caregiver spetta il compito di eseguire la medicazione indicata e gli obiettivi dati per ogni singolo stadio, avendo premura di rispettare anche gli allert.

Gli allert sono semplici consigli di buona pratica nella gestione delle lesioni, nei vari stadi. Sono indicati con un segno di "pericolo" proprio per creare nel destinatario l'attenzione a quegli aspetti che, se non appresi, sono fonte di errore.

Infine è stato inserito un paragrafo sulla gestione del dolore.

Si è visto, infatti, che queste ferite sono molto dolorose per i pazienti e il cambio della medicazione può diventare 
un momento difficile e traumatico per il soggetto. È giusto essere consapevoli che il dolore è una condizione comune

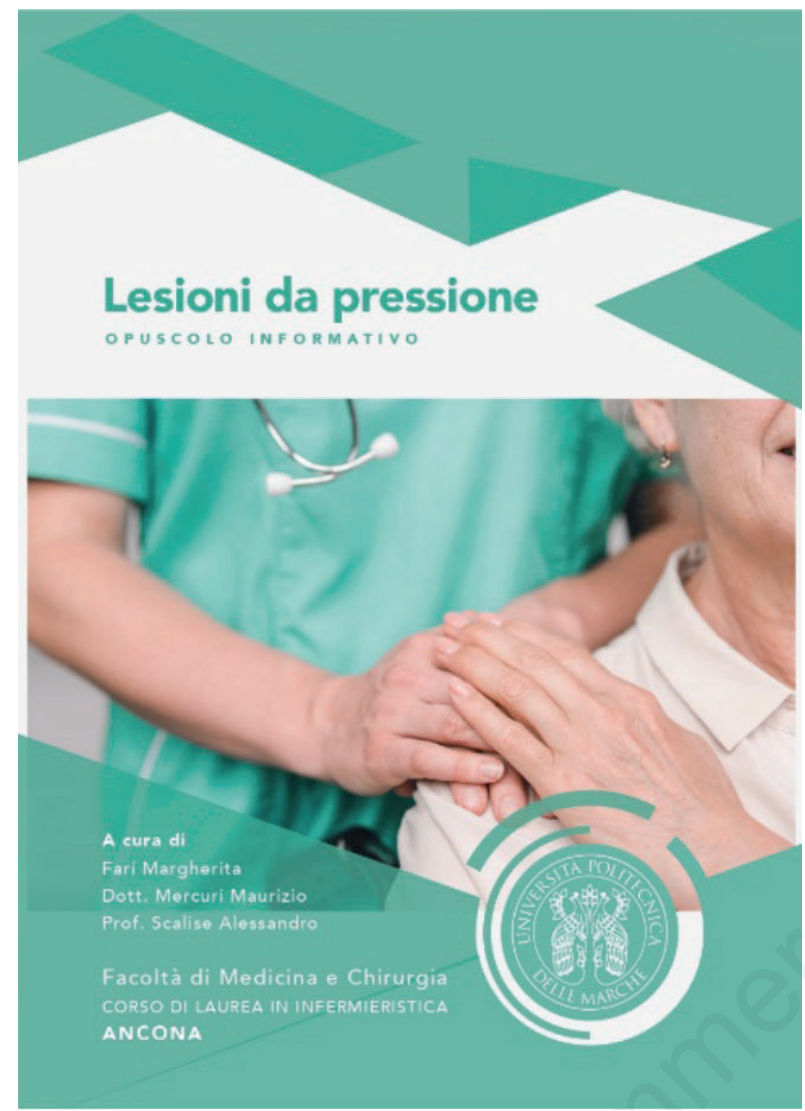

Figura 3. Copertina dell'opuscolo informativo per il cittadino. per questa tipologia di pazienti e che nel creare dolore non si sta sbagliando manovra, per questo sono state introdotte tecniche per cercare di alleviarlo.

Il caregiver non ha l'obiettivo di sostituire il professionista ma a questa figura viene chiesto di acquisire la consapevolezza della patologia e le conoscenze di base necessarie alla gestione di questa condizione cronica.

\section{CONCLUSIONI}

La gestione delle ferite difficili è garantita dalla presenza di professionisti qualificati. Molti operatori in possesso di master di primo livello lavorano in ambito territoriale per gestire questa condizione i cui numeri sono in ascesa. ${ }^{14}$

È da considerarsi però che la richiesta che viene fatta dai cittadini è maggiore rispetto ai servizi che possono garantire azienda e territorio.

La creazione dell'opuscolo informativo cerca di aiutare le famiglie dei pazienti con LDP che necessitano di medicazioni molto frequenti ed ai quali non può essere assicurata una valutazione specialistica frequente.

L'obiettivo è quindi quello di formare, anche se con nozioni base, il cittadino e istruirlo nella gestione al fine di collaborare con il territorio e l'azienda per la salvaguardia e il miglioramento della salute del paziente.

L'opuscolo non implica il completo distacco del cittadino nei confronti dell'azienda e del territorio, anzi, è stato creato per elaborare un sistema unito e competente.

I limiti di questo progetto, allo stato attuale, è la sua mancata diffusione agli utenti. Presentati gli obiettivi, non

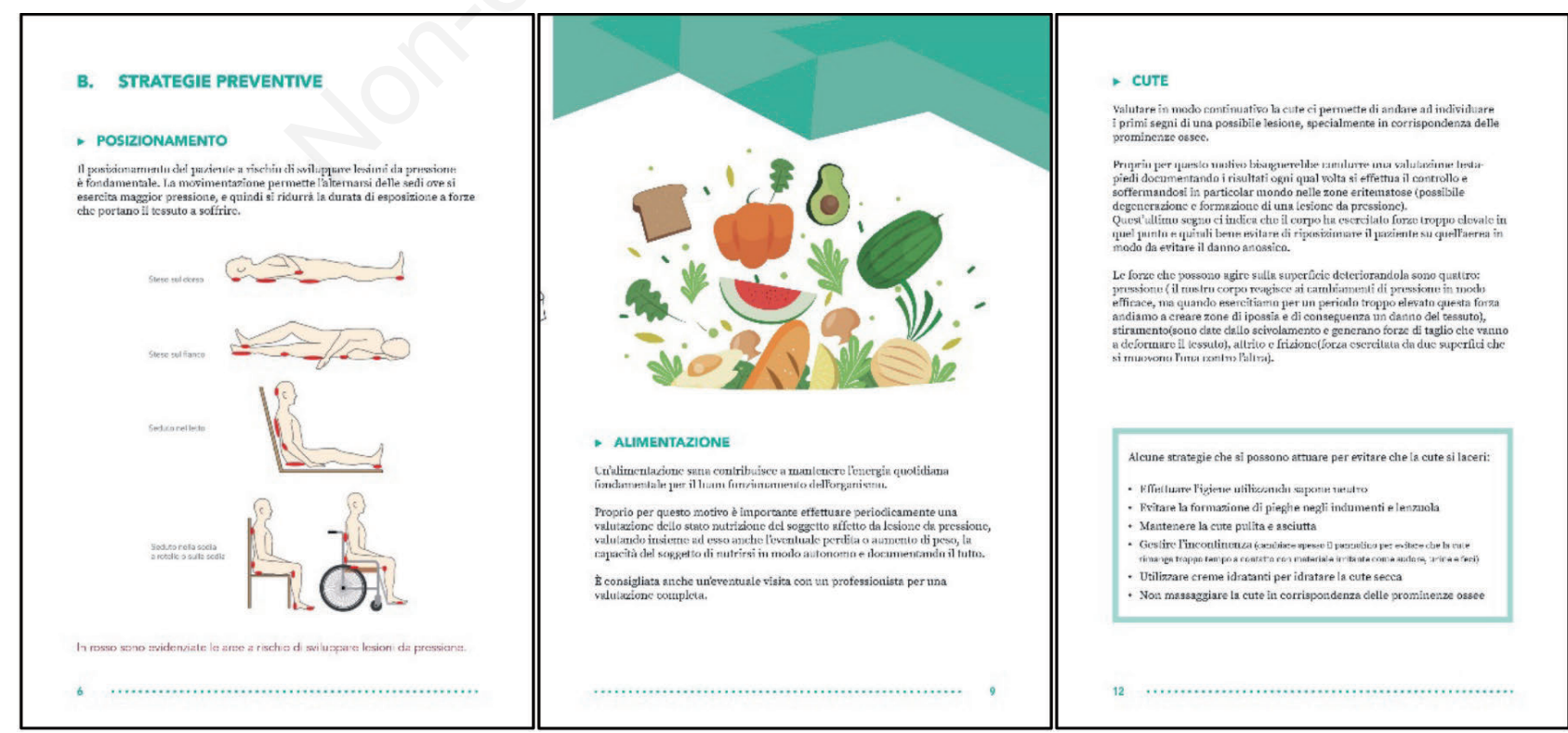

Figura 4. Le nozioni base per la vita quotidiana, tra cui la mobilizzazione, l'alimentazione e la cura della pelle, come discusse nell'opuscolo informativo. 


\section{SCALA DI BRADEN}

VALUTAZIONE DEL RISCHIO DI LESIONI DA PRESSIONE

Ad aiutarci nella valutazione di quelli che sono i soggetti a rischio di sviluppare lesioni da pressione possiamo utilizzare strumenti come la scala di Braclen. Consiste nellassegnare un punteggio in base alle condizioni del paziente per quanto riguarda un determinato ambito; la valutazione dovrebbe essere fatta appena si prende in carico il soggetto e ripetuta dopo 14 giorni nel momento in cui le condivioni del paviente si modifichino.

\begin{tabular}{|c|c|c|c|c|}
\hline \multirow[t]{2}{*}{ INDICATORI } & \multicolumn{4}{|c|}{ PUNTEGGIO } \\
\hline & 1 & 2 & 3 & 4 \\
\hline $\begin{array}{l}\text { PERCEZIONE } \\
\text { SENSORIALE }\end{array}$ & $\begin{array}{l}\text { IOLALMENIE } \\
\text { UMIATA }\end{array}$ & $\begin{array}{l}\text { MOLIO } \\
\text { UMTIAAA }\end{array}$ & $\begin{array}{l}\text { LEGCERMMENIE } \\
\text { LMIIAAA }\end{array}$ & $\begin{array}{l}\text { NON } \\
\text { UMITATA } \\
\end{array}$ \\
\hline MACERAZIONE & $\begin{array}{l}\text { COSTANTEMENTE } \\
\text { UMMA }\end{array}$ & $\begin{array}{l}\text { MOLTO } \\
\text { WMIDA }\end{array}$ & $\begin{array}{l}\text { OCCASIONAIMENTE } \\
\text { UMDA }\end{array}$ & $\begin{array}{l}\text { RARAMENTE } \\
\text { UMIDA }\end{array}$ \\
\hline ATTIVITA & ALEIIAIO & IN POURONA & $\begin{array}{l}\text { CAMMINAA } \\
\text { OCCASIONAIMENTC }\end{array}$ & $\begin{array}{l}\text { CAMMINA } \\
\text { SPESSO }\end{array}$ \\
\hline MOBILITA & $\begin{array}{l}\text { COMPLETAMENTE } \\
\text { IMMOELE }\end{array}$ & $\begin{array}{l}\text { MOLTO } \\
\text { UMITATA }\end{array}$ & $\begin{array}{l}\text { PARZINLMENTE } \\
\text { LIMIIATA }\end{array}$ & $\begin{array}{l}\text { NON } \\
\text { UMTIATA }\end{array}$ \\
\hline NUTRIZIONE & NOLTO POVERA & $\begin{array}{l}\text { PROBABII MINNT } \\
\text { rOVERA }\end{array}$ & NDEGUATA & OTIMMLE \\
\hline $\begin{array}{l}\text { FRIZIONE E } \\
\text { SCIVOLAMENTO }\end{array}$ & $\begin{array}{l}\text { PROHHAMA } \\
\text { PRESENAIE }\end{array}$ & $\begin{array}{l}\text { PROHEMA } \\
\text { POTENZ/ALE }\end{array}$ & $\begin{array}{l}\text { PrizOSI MAA } \\
\text { ASSERTE }\end{array}$ & $\mathbf{x}$ \\
\hline
\end{tabular}

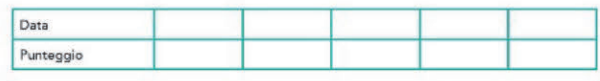

\begin{tabular}{|l|l|}
\hline$<16$ & Grave rischio di compromissione dell lintegrita cutanea \\
\hline
\end{tabular}

$=16 \quad$ Rischio di compromissione dell' integrità cutanea

\begin{tabular}{ll} 
da 16 a 18 Lieve rischio di compromissione dellintegrità cutanea \\
\hline
\end{tabular}

\begin{tabular}{l|l}
\hline$>18$ & Non presenta rischi di compromissione dell'integrita cutanes
\end{tabular}
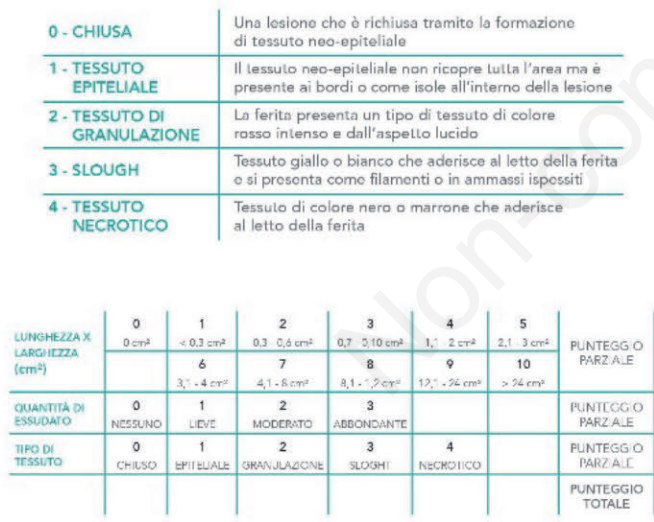

\begin{tabular}{|l|l|l|l|l|l|}
\hline Dato & & & & & \\
\hline Punteggio & & & & & \\
\hline
\end{tabular}

Figura 5. Le scale di valutazione, quali Braden e Push tool, nell'opuscolo informativo.

abbiamo dati sulla sua efficacia e praticità per mancanza di riscontro.

Questo opuscolo viene presentato come una proposta per il territorio e l'azienda, con la speranza che la sua applicazione porti ai risultati desiderati.

$\mathrm{Ci}$ si aspetta, in futuro, la possibilità di validare l'opuscolo tramite esperti e di esportarlo a livello territoriale tramite la collaborazione degli ambulatori, addetti al trattamento delle ferite difficili.

\section{B. MEDICAZIONE}

- 10 STADIO

\begin{tabular}{|c|c|c|}
\hline OBIETTIVI & INTERVENTO & DURATA \\
\hline \multirow{2}{*}{$\begin{array}{l}\text { PREVENIRE LA } \\
\text { DEGENERAZIONE } \\
\text { DELLA LESIONE }\end{array}$} & \multirow{2}{*}{$\begin{array}{l}\text { RIPOSIZIONARE } \\
\text { IL PAZIENTE }\end{array}$} & $\begin{array}{l}\text { OGNI } 12 \text { ORE } \\
\text { SE ALLETAATO }\end{array}$ \\
\hline & & $\begin{array}{l}\text { OGNI } 20 \text { MINUTI } \\
\text { SE IN CARROZZINA }\end{array}$ \\
\hline \multirow{2}{*}{$\begin{array}{l}\text { SALVAGUARDARE } \\
\text { LA CUTE }\end{array}$} & CREMA & \\
\hline & UNGUENTO & \\
\hline \multirow{3}{*}{$\begin{array}{l}\text { AUMENTARE } \\
\text { LA PROTEZIONE }\end{array}$} & IDROCOLLOIDE & 7 GIORNI \\
\hline & $\begin{array}{l}\text { IDROCOLLOIDE } \\
\text { EXTRASOTTILE }\end{array}$ & 7 GIORNI \\
\hline & $\begin{array}{l}\text { SCHIUMA IN } \\
\text { POLIURETANO } \\
\end{array}$ & 7 GIORNI \\
\hline RIDURRE FRIZIONE & $\begin{array}{l}\text { FILM SEMIPERMEABILE } \\
\text { TRASPARENTE }\end{array}$ & 7 GIORNI \\
\hline
\end{tabular}

Collagene: es. CONDRESS, BIONECT Idrocolloidi: es. COMFEEL, DUODERM
Schiuma di poliuretano: Es. BIATAIN, ALLEYYN Schiuma di poliuretano: es. BIATAIN, ALLEVYN

! NON MASSAGGIARE LA CUTE VIGOROSAMENTE MENTRE SI APPLICANO I PRODOTI

Figura 6. Esempio di tabella con le medicazioni avanzate considerate più idonee, per i singoli stadi.

\section{BIBLIOGRAFIA}

1. Annuario Statistico Italiano. [S.L.]: Ist Naz Di Statistica; 2019.

2. Weiten W. 1950-, Psychology applied to modern life: adjustment in the 21 st century. 9th ed. Wadsworth Cengage Learning: 2009.

3. Commissione Regionale Dispositivi Medici, Direzione Generale Sanità e Politiche Sociali e per l'Integrazione. Le medicazioni avanzate per il trattamento delle ferite acute e croniche. Allegato 1: Protocollo di trattamento delle lesioni da pressione. Servizio Assistenziale Territoriale - Area Farmaco e Dispositivi Medici, gennaio 2016. 
4. Marazzi M, Falcone L. Le Ferite Difficili: Linee Guida Di Trattamento. 2016.

5. Demo.istat.it. 2020. Demo-Geodemo. - Mappe, Popolazione, Statistiche Demografiche Dell'istat. Visualizzato 6 Aprile 2020. Disponibile presso: http://demo.istat.it/altridati/ previsioni_naz/

6. Regolamento Regionale 18 gennaio 2007, n. 4. Regolamento Regionale attuativo della legge regionale 10 luglio 2006, n. 19.

7. FNOPI. Master Specialistici delle Professioni Sanitarie 2020. Visitato il 25 Marzo 2020. Disponibile presso: https://www. fnopi.it/wp-content/uploads/2019/10/ELENCOCOMPLETO-MASTER.pdf

8. Scalise A. Lesioni Cutanee Croniche. Milano: Edra; 2015.

9. European Pressure Ulcer Advisory Panel, National Pressure Injury Advisory Panel and Pan Pacific Pressure Injury Alliance. Prevention and Treatment of Pressure Ulcers/Injuries: Quick Reference Guide. Emily Heasler (Ed.). EPUAP/ NPIAP/PPPIA: 2019.

10. National Pressure Ulcer Advisory Panal, European Pressure
Ulcere Advisor Panal and Pan Pacific Pressure Injury Alliance. Prevencion and Treatment of Pressure Ulcers: Quick Reference Guide. Emily Haesler (Ed.). Cambridge Media: Osborne Park Australia; 2014.

11. Commissione Regionale Dispositivi Medici, Direzione Generale Sanità e Politiche Sociali e per l'Integrazione. Le medicazioni avanzate per il trattamento delle ferite acute e croniche. Allegato 4: Scale di valutazione delle lesioni cutanee. Servizio Assistenziale Territoriale - Area Farmaco e Dispositivi medici, gennaio 2016.

12. Bonadeo P, Marazzi M. Wound Bed Preparation: Evoluzione della Pratica Clinica Secondo I Principi Del TIME. Milano: Aretrè; 2004.

13. Carnali M, Nicolini M, Paolinelli M, et al. Procedura AV2. Prevenzione e cure lesioni da pressione. Professioni sanitarie area infermieristico-ostetricia AV2.

14. Legge 1 Febbraio 2006, n.43. Disposizioni in materia di professioni sanitarie infermieristiche, ostetrica, riabilitative, tecnico-sanitarie e della prevenzione e delega al governo per l'istituzione dei relativi ordini professionali. 